\title{
Ventricular hypertrophy in cardiomyopathy
}

\author{
Celia Oakley \\ From the Royal Postgraduate Medical School, Hammersmith Hospital, London W.12
}

Semantic difficulties arise when hypertrophic obstructive cardiomyopathy is seen without obstruction and with congestive failure, and also when congestive cardiomyopathy is seen with gross hypertrophy but without heart failure. Retention of a small left ventricular cavity and a normal ejection fraction characterizes hypertrophic cardiomyopathy at all stages of the disorder. Congestive cardiomyopathy is recognized by the presence of a dilated left ventricular cavity and reduced ejection fraction regardless of the amount of hypertrophy and the presence or not of heart failure.

Longevity in congestive cardiomyopathy seems to be promoted when hypertrophy is great relative to the amount of pump failure as measured by increase in cavity size. Conversely, death in hypertrophic cardiomyopathy is most likely when hypertrophy is greatest at a time when outflow tract obstruction has been replaced by inflow restriction caused by diminishing ventricular distensibility. Hypertrophy is thus beneficial and compensatory in congestive cardiomyopathy, whereas it may be the primary disorder and eventual cause of death in hypertrophic cardiomyopathy.

Reasons are given for believing that hypertension may have been the original cause of left ventricular dilatation in some cases of congestive cardiomyopathy in which loss of stroke output thenceforward is followed by normotension. Development of severe hypertension in these patients after recovery from a prolonged period of left ventricular failure with normotension lends weight to this hypothesis.

No fault has been found in the large or small coronary arteries in either hypertrophic cardiomyopathy or congestive cardiomyopathy when they have been examined in life by selective coronary angiography, or by histological methods in biopsy or post-mortem material. Coronary blood supply may be a limiting factor in the compensatory hypertrophy of congestive cardiomyopathy, and the ability to hypertrophy may explain the better prognosis of some patients. In hypertrophic cardiomyopathy excessive metabolic demand may not be met, and inadequacy of blood flow may contribute both to sudden death and to progressive replacement fibrosis in this disease.

Histochemical and ultrastructural methods have failed to show any fundamental differences between hypertrophic cardiomyopathy and congestive cardiomyopathy, whereas conventional histology permits recognition of hypertrophic cardiomyopathy and distinction both from congestive cardiomyopathy and from 'normal' secondary hypertrophy in organic aortic stenosis.

Cardiomyopathies have been divided into three main groups: congestive, hypertrophic, and obliterative (Goodwin, 1970). Left ventricular hypertrophy is the most prominent feature of both the congestive and the hypertrophic cardiomyopathies.

Congestive cardiomyopathies have left ventricular dilatation as their cardinal feature, and are so named because of the usual development of congestive cardiac failure at some stage in the disease. Hypertrophy of the left ventricular wall is commonly present and may reach considerable proportions in longsurviving cases (Fig. I).

Hypertrophic cardiomyopathies have left 13 ventricular hypertrophy as their outstanding feature, but are distinguished from congestive cardiomyopathies by absence of left ventricular dilatation. A hypertrophied but undilated left ventricle is therefore the hallmark of hypertrophic cardiomyopathy. This remains true at all stages of the disease, and the left ventricle does not dilate even after the development of clinical heart failure. Obstruction to the outflow of blood from the left ventricle commonly occurs, but appears not to be persistent throughout the natural history of the disorder (Goodwin and Oakley, 1970) (Fig. 2).

The obliterative cardiomyopathies include 
endomyocardial fibrosis and Loeffler's endomyocarditis, in which ventricular cavity obliteration and atrioventricular valve incompetence are associated with slight or absent wall hypertrophy. They will not be further considered here.

In congestive cardiomyopathy some ventricular hypertrophy is always present, but the least hypertrophy is seen in the hearts of patients who have died quickly after only a short illness which is usually regarded as a myocarditis. Hypertrophy develops only with continuance of ventricular pump failure, and its absence in cases of rapid death testifies to the recent onset of the fatal cardiac disorder. The link between such fulminating myocarditis and chronic congestive cardiomyopathy is tenuous, and patients have only rarely been followed from the acute illness into the chronic one.

Leaving aside certain specific myocardial infiltrations such as haemochromatosis and amyloid, ventricular hypertrophy in congestive cardiomyopathy seems to be related both to the severity of the functional impairment and to its duration. In patients with longstanding congestive failure left ventricular hypertrophy is sometimes gross and the heart may reach more than one kilo in weight. Such very heavy hearts are rarely seen in 'hypertrophic' cardiomyopathy, and the extent of the hypertrophy in some cases of congestive cardiomyopathy has led to semantic confusion. The terms 'idiopathic hypertrophy' and 'idiopathic cardiomegaly' are also used to describe such hearts. When the hypertrophied wall surrounds a dilated left ventricular cavity the patient has failure of systolic pump function with an increased end-diastolic volume and reduced ejection fraction - congestive cardiomyopathy. When the hypertrophied wall surrounds a cavity of normal or reduced size the patient obviously retains a normal or increased systolic ejection fraction. These cases are properly described as hypertrophic cardiomyopathies, and evidence which suggests that these are at all stages of the disease fundamentally different from the congestive cardiomyopathies will be presented later in the paper.

\section{Relation between hypertrophy and prog-} nosis in congestive cardiomyopathy

Returning now to the congestive cardiomyopathies of unknown aetiology, the relation between the amount of hypertrophy, the severity of the functional impairment, the duration of the disease, the prognosis, and the existence of concurrent or antecedent hypertension has been studied by Drs. Croxson,
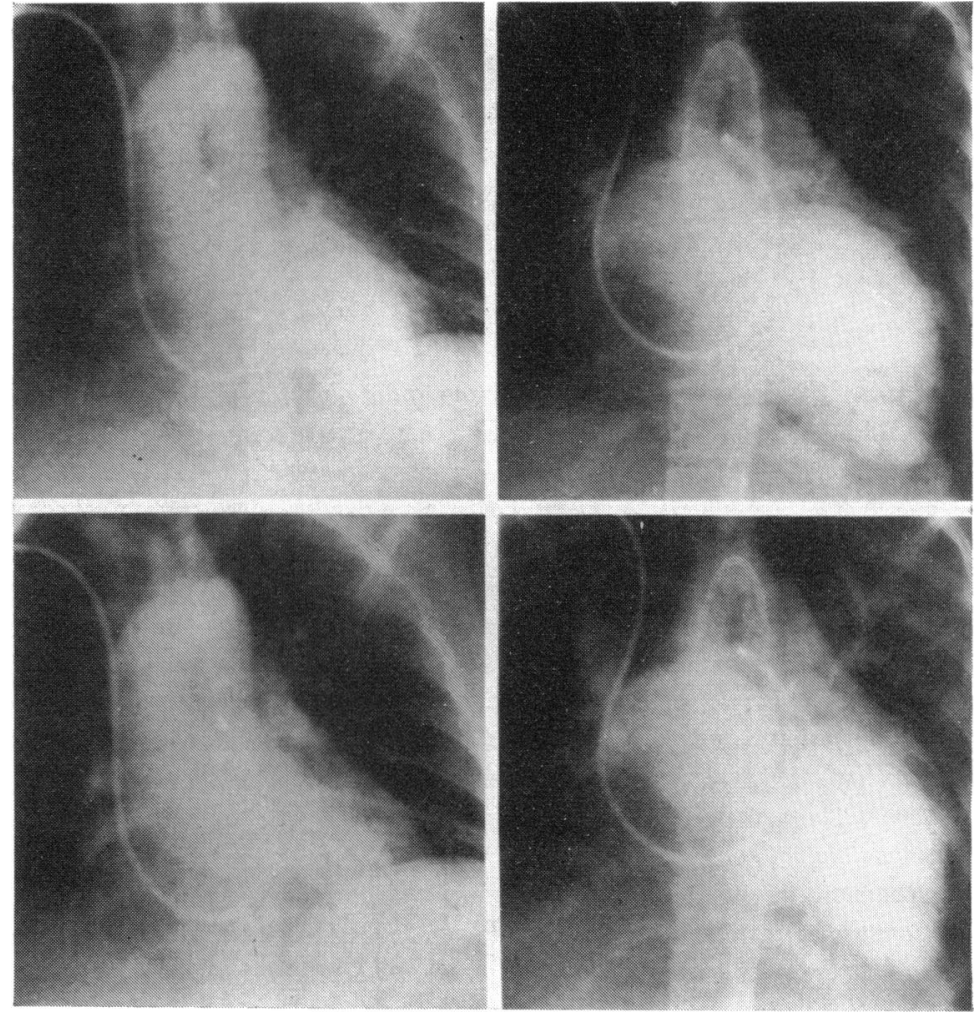

FIG. I Frontal view angiocardiograms showing the left ventricle opacified after contrast injection into the pulmonary artery in two patients with congestive cardiomyopathy. Diastolic frames are above and systolic below. The frames on the right are from a patient who died a few months after the study. The cavity of the left ventricle is so dilated that the eye can discern little difference between systole and diastole, but the wall of the ventricle is only slightly thickened. The patient whose angiograms are shown on the left was far less sick. The cavity of the left ventricle is less dilated but its wall is thicker than is the case in the other patient.

Kristinsson, and Raphael at the Royal Postgraduate Medical School, London (Croxson and Raphael, 1969; Kristinsson, 1969). Left ventricular volumes have been measured by a modification of Arvidsson's angiographic method (I96I), and left ventricular mass has been calculated by Rackley et al.'s method (1964). The end-diastolic volumes, ejection fractions, ventricular mass, and the ratio between wall thickness and the maximal cavity size in diastole were measured (Fig. 3). Survivors were compared both with those who had died since being studied and with 
patients without disorders of the left ventricle who were undergoing diagnostic catheterization but who were found either to have abnormalities which only affected the right ventricle or normal hearts.

Prolonged survival was unusual when the absolute end-diastolic volume exceeded 200 $\mathrm{ml} . / \mathrm{m} .^{2}$ or $2 \frac{1}{4}$ times the maximal enddiastolic volume found among the normal controls. Prolonged survival was unusual when the ejection fraction was less than 10 per cent compared with over 60 per cent in the normal controls. There was no correlation between the absolute left ventricular muscle mass or wall thickness and prolonged survival, but survival was also unusual when the diastolic width of the cavity divided by the thickness of the wall exceeded 8 even though the end-diastolic volume was still well under $200 \mathrm{ml} . / \mathrm{m}^{2}$

A longer survival time in congestive cardiomyopathy was thus found to be associated with the presence of adequate 'relative hypertrophy' - that is, hypertrophy whose amount seemed to be proportional to the severity of the left ventricular functional impairment. Nevertheless no amount of hypertrophy seemed to permit survival once a certain stage of left ventricular dilatation had been reached. A longer known duration of illness was associated with a greater left ventricular mass, but a greater left ventricular mass per se gave no clue to future prognosis. Thus death in congestive cardiomyopathy may be related to the ability of the heart to hypertrophy, as well as to the severity of the functional impairment.

Hypertrophy may counteract the tendency to progressive dilatation, but loss of ventricular distensibility leads to a rise in left ventricular end-diastolic pressure which may be an important stimulus to further hypertrophy. It may be asked whether hypertrophy which was originally compensatory ever becomes an impediment by causing an increase in metabolic needs beyond the capacity of the coronary system to meet them. The height of the left ventricular diastolic pressure showed no correlation with the extent of the left ventricular dilatation and no adverse correlation with prognosis. Though the end-diastolic pressure was usually high in the patients with very big left ventricular cavities, it was often equally high in patients with successful 'relative hypertrophy', and the left ventricular filling pressure thus showed more correlation with an increased left ventricular muscle mass than with the extent of functional impairment in the sense of failure of systolic ejection.

Unfortunately from these studies we gain no knowledge of the total duration of disease.
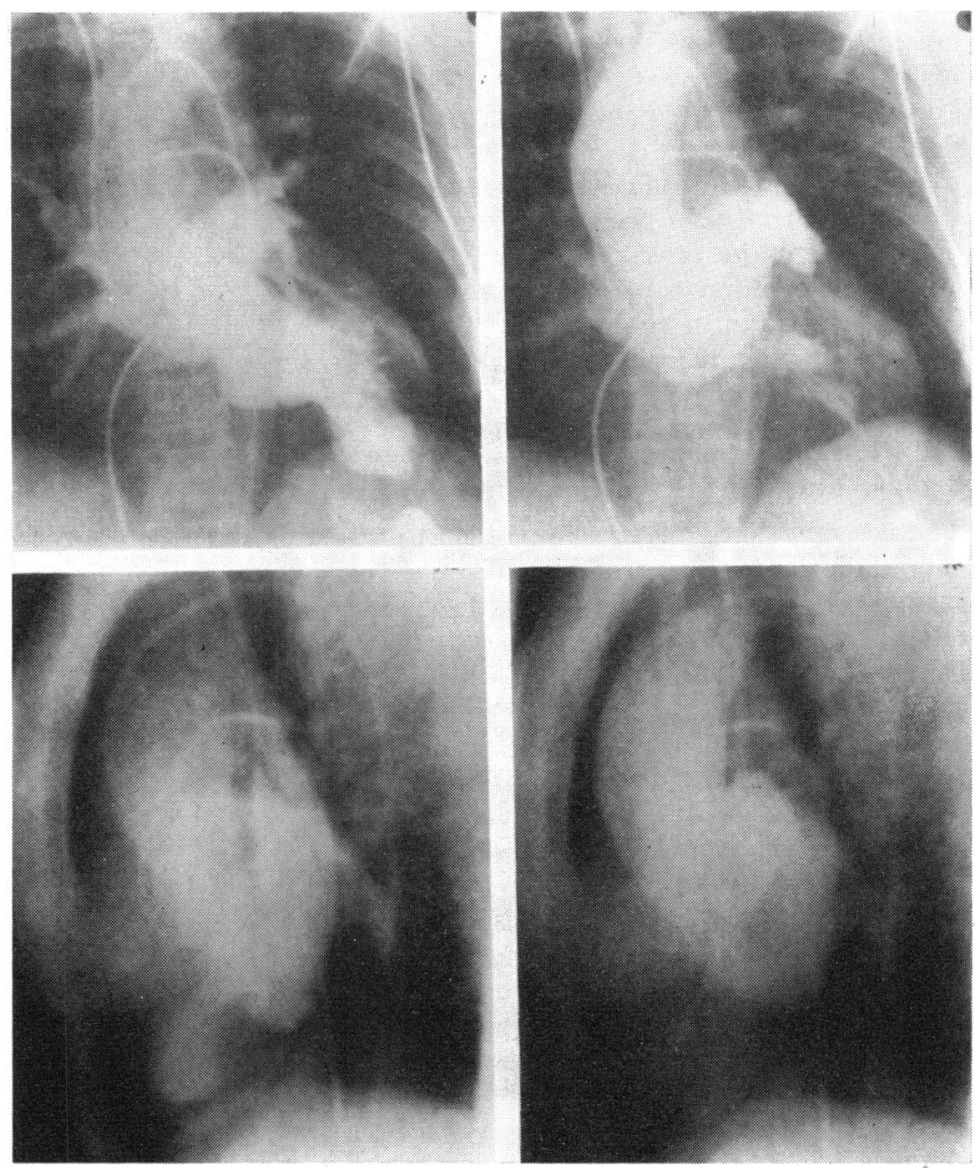

FIG. 2 Left atrial injection of contrast for left ventricular volume study in a patient with hypertrophic obstructive cardiomyopathy shows a small dilated cavity and an extremely thick wall. The left-hand frames were taken during early systole and show the encroachment of the papillary muscles upon the cavity. The right-hand frames were taken in endsystole and show the almost empty ventricle at the completion of ejection.

Some patients were first recognized because of symptoms and some because of an abnormal chest radiograph, or because they presented to the doctor with disorder in another organ-system and heart disease was an incidental finding. Once cardiac abnormality was known, symptoms were probably both more likely to be voiced and more likely to be attributed to the heart. The timing of the study therefore depended on the accident of when they were seen, and this depended on many things other than the severity of their disease. Death subsequent to the study may depend to some extent upon how early they 
came in the series as well as upon the prognostic importance of the various parameters which were being assessed. We had no knowledge of the gradient of their deterioration in most instances.

As some of the biggest and most hypertrophied hearts have been found among the patients with the fewest symptoms and the least deterioration since diagnosis, hypertrophy seems not to be detrimental but compensatory in the majority of patients.

\section{Relation between hypertensive heart disease and congestive cardiomyopathy}

The relevance of a high systemic blood pressure to the genesis and prognosis of congestive cardiomyopathy is hard to elucidate. Sustained hypertension was present in I I out of 80 congestive cardiomyopathies which have been studied at the Royal Postgraduate Medical School. Sustained hypertension has developed since study in three other patients who presented with left ventricular failure, and in whom a diagnosis of congestive cardiomyopathy was made before it was relinquished in favour of a diagnosis of hypertensive heart disease. Many patients with severely hypertrophied left ventricles as judged clinically, radiologically, and electrocardiographically have mildly elevated blood pressures which do not seem to be raised sufficiently to account for the left ventricular abnormality. They differ only in being hypertensive from the patients with congestive cardiomyopathy and normal blood pressures. Similarly, patients with congestive cardiomyopathy differ only in their normal blood pressures from the hypertensives with severe left ventricular disorder in whom a diagnosis of congestive cardiomyopathy would seem to be ridiculous. The left ventricular functional disorder and subsequent pathology are similar, and the failing hypertrophied left ventricle apparently only has one appearance whether it is examined by conventional light microscopy or by more sophisticated electron microscopy and histochemical analysis. No aetiological clue is given, and the more refined the test the more disappointing the difference seems to be between one fragment of hypertrophied myocardium and another.

In patients with functional stability, few symptoms, and considerable left ventricular hypertrophy, a case can be made for regarding them as having hypertensive heart disease whatever the blood pressure. Acute afterloading of the normal left ventricle by the administration of an $\alpha$-stimulating vasopressor drug which causes a sudden rise in
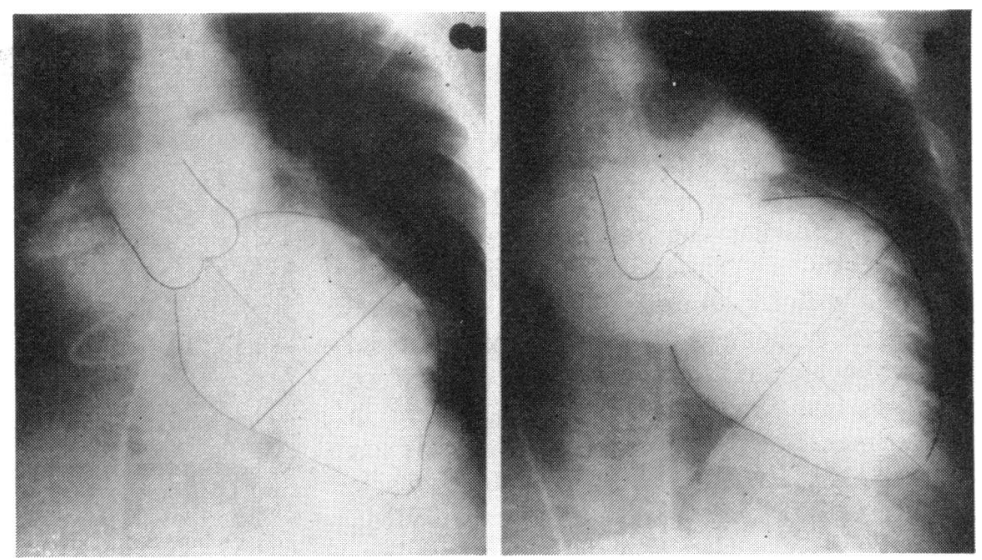

FIG. 3 Left atrial contrast injection in severe congestive cardiomyopathy showing the change in dimensions between systole on the left and diastole on the right. The wall thickness at the point of measurement is also shown.

arterial pressure causes a fall in ejection fraction and a rise in end-diastolic volume and pressure (left ventricular failure) which is reversible on removing the cause. Rapidly developing hypertension from sudden renal ischaemia or other causes may lead to such left ventricular failure. If the stroke output falls the blood pressure will also fall unless the vasoconstriction increases. It is suggested that if the rise in peripheral vascular impedance which caused the left ventricle to fail remains unchanged the heart may either recover through the development of hypertrophy or remain dilated despite the development of hypertrophy and the blood pressure may accordingly either rise again (hypertensive heart disease) or remain within normal limits (congestive cardiomyopathy).

Primary or secondary genesis of hypertrophy in hypertrophic cardiomyopathy

In hypertrophic obstructive cardiomyopathy it has been suggested that the hypertrophy is secondary to outflow tract obstruction and that the outflow tract obstruction may be due to some incoordination of ventricular contraction. Since obstruction to left ventricular ejection is frequently mild or even absent in the hearts which show the greatest amount of hypertrophy, and since the gradient develops only after the left ventricle has already freely ejected 50 per cent or more of its contents, this thesis seems untenable. It has been 
suggested that the mitral regurgitation which is nearly always present in those patients who have outflow obstruction itself engenders outflow tract occlusion by reducing left ventricular cavity size prematurely during ejection (Oakley et al., 1967). Since the mitral regurgitation is mainly a late systolic event similar in timing to the outflow obstruction, this explanation also seems to be inadequate, but the abnormality of the mitral valve apparatus which is responsible for mitral reflux may cause outflow tract obstruction through another mechanism. The premature excitation or abnormal architecture of the anterior papillary muscle may be responsible both for the reflux and for the movement of the anterior cusp across the outflow tract to impinge on the ventricular septum during ventricular ejection in these patients (Fig. 4). This movement can be seen both on cineangiography and on echocardiographic recordings from the anterior mitral cusp (Pridie and Oakley, 1970).

The flaw in this argument is that many patients with hypertrophic cardiomyopathy spontaneously lose both outflow obstruction and mitral regurgitation when watched over the course of years, but the hypertrophy does not regress. It seems rather to progress and a climbing left ventricular end-diastolic pressure betrays a progressive deterioration in left ventricular compliance. Since a normal ejection fraction is maintained and the left ventricular cavity does not become dilated, this progressive hypertrophy now occurring in the absence of obstruction is not compensatory. It is the hypertrophy and associated fibrosis which alone provide the reason for the raised left ventricular filling pressure in hypertrophic cardiomyopathy.

Difference between hypertrophic cardiomyopathy without obstruction and congestive cardiomyopathy with much hypertrophy

The fundamental difference between the hypertrophic and congestive cardiomyopathies is emphasized by their differing responses to beta-adrenergic blockade and to sympathetic stimulation (Webb-Peploe, Croxson, and Oakley, 1970). Drs. Webb-Peploe and Croxson at the Royal Postgraduate Medical School have shown that beta-adrenergic blockade with practolol ${ }^{1}$ lowers the raised left ventricular end-diastolic pressure in hypertrophic

\footnotetext{
${ }^{1}$ Practolol ICI 50172, now called Eraldin.
}

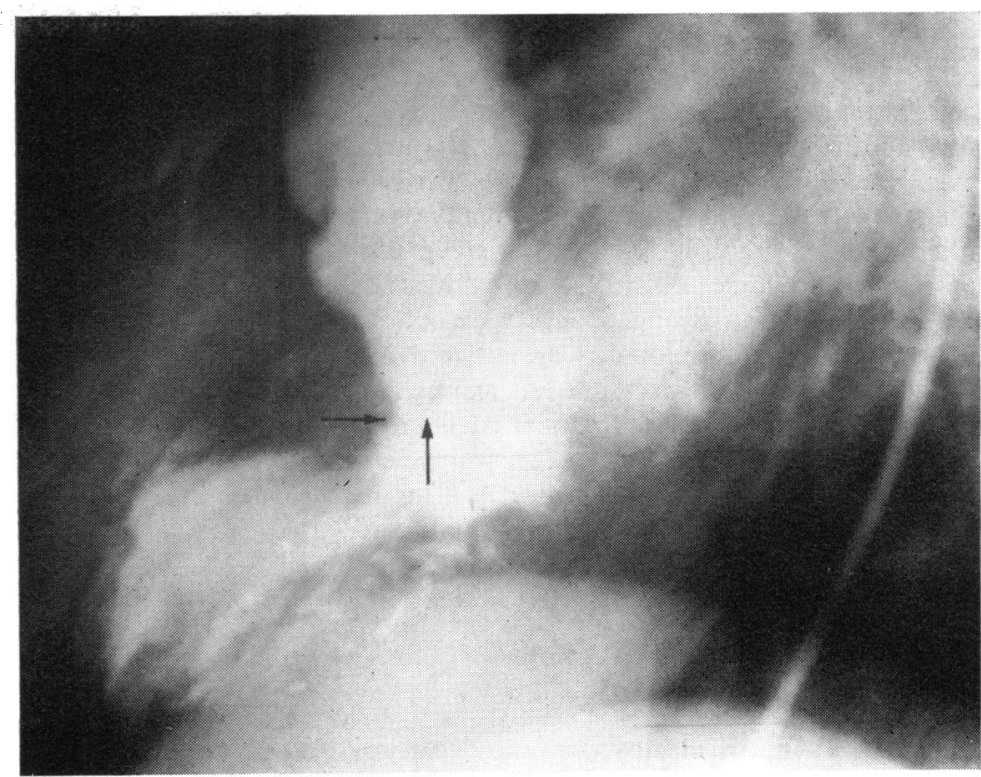

FIG. 4 Retrograde left ventricular angiogram, left lateral view, in hypertrophic obstructive cardiomyopathy shows the site of the outflow tract gradient when the anterior mitral cusp moves over to impinge on the septum. Mitral regurgitation is seen.

cardiomyopathy without reducing left ventricular stroke work. Since the left ventricular volume is not altered, this fall in end-diastolic pressure denotes an increase in the compliance of the ventricle and is analogous to the increase in the compliance of the normal dog left ventricle which has been demonstrated after total autonomic denervation. Conversely, betaadrenergic blockade causes the raised enddiastolic pressure in congestive cardiomyopathy to rise further. The same effect is seen in left ventricular failure associated with ischaemic heart disease. This is because the left ventricle which is failing to eject dilates further after removal of sympathetic inotropic stimulation. The difference in left ventricular cavity size and in systolic ejection fraction between the hypertrophied left ventricle in socalled congestive cardiomyopathy and the hypertrophied left ventricle in so-called hypertrophic cardiomyopathy is therefore seen to become the essential difference between the two and the final criterion for diagnosis.

This essential difference between the two groups is illustrated more simply by looking at the filling characteristics of the left ventricle 
by means of reflected ultrasound. The rate of diastolic closure of the anterior mitral cusp reflects the rate of ventricular distension. In congestive cardiomyopathy the left ventricle fills abnormally fast from a high left atrial pressure, but only for a short time, because the stroke volume is low and the already overfilled ventricle rapidly reaches the steep part of its compliance curve so the diastolic pressure rises and filling abruptly ceases. In hypertrophic cardiomyopathy the left ventricle also fills from a high pressure left atrium but the left ventricle contains a normal residual volume. As the distensibility of this muscle is low, filling rapidly slows off shortly after its commencement, but slow filling then continues throughout diastole (Fig. 5).

Coronary insufficiency in cardiomyopathy, coronary artery disease mimicking cardiomyopathy, and cardiomyopathy mimicking coronary artery disease

The possibility of coronary failure was looked at in both groups of cardiomyopathies. Anginal pain is not uncommon, particularly in the hypertrophic group. Interstitial fibrosis between the hypertrophied muscle fibres is a variable feature in both groups, but it is greatest in patients who have survived longer since diagnosis, in older patients, and in the patients with the heaviest hearts. Not surprisingly, too, greater distortion of the electrocardiogram is found in those with the heaviest hearts, longest duration of known disorder, and most severe dysfunction. In hypertrophic cardiomyopathy abnormal $Q$ waves in anterior praecordial leads together with an abnormally leftward mean frontal axis (left anterior hemiblock) are not uncommon and have been ascribed to septal fibrosis by Wigle (1964) and to a change in spatial orientation of the septum by Coyne (1968). In congestive cardiomyopathy focal abnormalities with $Q$ waves simulating infarction are also not uncommon (Gau et al., 1970; Raftery, Banks, and Oram, 1969). In the older patients who also had angina, coronary artery disease was often the diagnosis with which they had been referred to us. Anginal pain and an electrocardiogram suggestive of focal infarction have not yet been seen in congestive cardiomyopathy except in patients with overtly severe left ventricular dysfunction or hypertrophy, and the possibility of coronary failure from excess of demand over normal supply has seemed real. Selective coronary angiography and left ventricular cine-angiography were accordingly

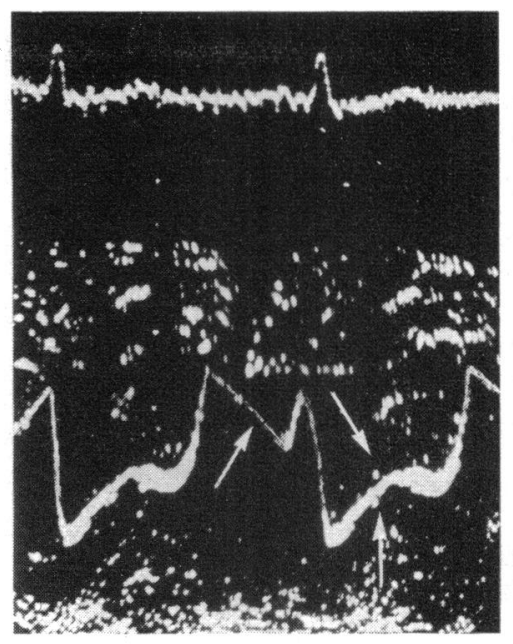

FIG. $5 a$

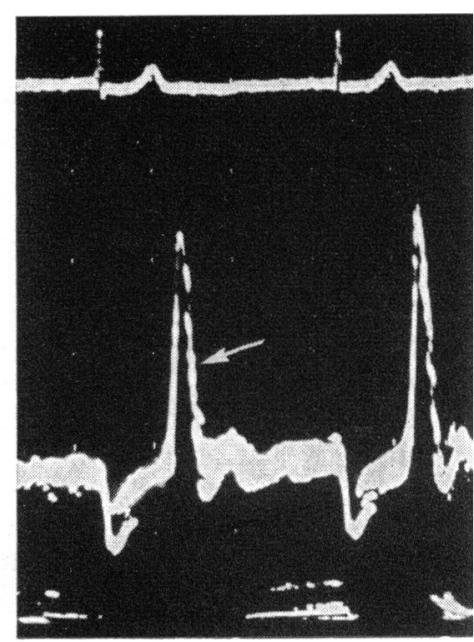

FIG. 5 b
FIG. 5a Mitral echocardiogram in hypertrophic cardiomyopathy with left ventricular obstruction and mitral reflux.

Downward deflections are closing movements and upward deflections are opening movements of the anterior cusp. The record shows

(I) systolic reopening of the valve; (2) slow diastolic distension of the ventricle indicated by a slow diastolic closure slope; (3) the close proximity of septal echoes to the anterior cusp, particularly during the systolic reopening movement when the cusp actually meets the septum.

FIG. 5b Mitral regurgitation in congestive cardiomyopathy with functional mitral reflux shows (I) very rapid ventricular filling with very early cessation of filling and return of the valve to the intermediate position; (2) no systolic reopening movement; (3) no septal echoes.

carried out at the Royal Postgraduate Medical School by Drs. Gau and Raphael in 8 patients with hypertrophic cardiomyopathy, of whom 5 had an infarct pattern on the electrocardiogram. Five out of 30 patients with congestive cardiomyopathy had an infarct pattern on the electrocardiogram; 14 of these were examined by selective coronary angiography and 16 at necropsy (Fig. 6). No coronary abnormality, focal wall thinning, or focal ventricular asynergy was recognized in any of these patients. No focal area of necrosis, fibrosis, or even of particularly severe myocardial change was found (Gau et al., 1969). 
FIG. 6a Electrocardiogram from a patient with congestive cardiomyopathy showing pseudoinfarction. The record would conventionally be reported as showing anteroseptal infarction with left anterior hemiblock.

FIG. 6b Selective contrast injection into the right coronary artery in the same patient. There is an area of obvious disease and narrowing, but good filling beyond and no collateral.
FIG. 6c Normal left coronary artery in the same patient in whom necropsy revealed no focal necrosis or fibrosis and no coronary artery disease except for the one area of nonocclusive atheroma in the right coronary artery which had been visualized.
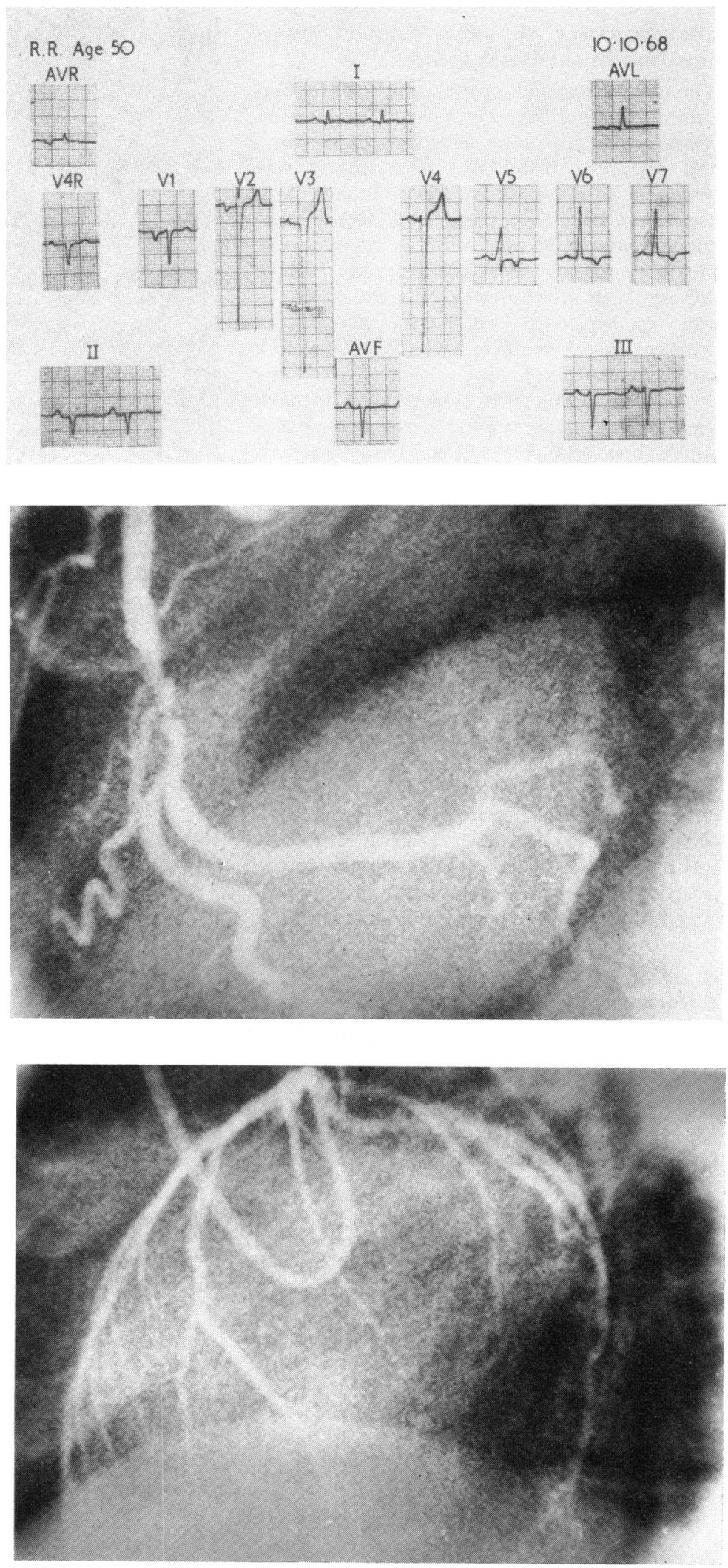
Study of histology, histochemistry, and ultrastructure of hypertrophied myocardium in cardiomyopathy

The hypertrophied myocardium has been submitted to a battery of investigations by conventional histology, electron microscopy, and chemical histology. The muscle was obtained by open biopsy from patients with congestive cardiomyopathy and during ventriculomyotomy in patients with hypertrophic cardiomyopathy. That the hearts behave differently in life at all phases of the disease is now established. The hearts look quite different in the hand as well, the congestive cardiomyopathies having a dilated left ventricle with symmetrical hypertrophy in contrast to the hypertrophic cardiomyopathies which show small left ventricular cavities with hypertrophy which is much more conspicuous in the interventricular septum than in the free wall. Light microscopy reveals varying amounts of fibrosis in both, but the hypertrophied muscle fibres are shorter and thicker in the hypertrophics and the arrangement of the muscle fibres often shows characteristic whorling which is absent in the congestives, in which the hypertrophied myocardial fibres resemble more closely 'normal' hypertrophied muscle obtained from patients with organic aortic stenosis (Fig. 7). Once electron microscopy and histochemical techniques are employed these differences become marginal (Kristinsson et al., 1969). It is indeed frustrating that the more refined the analysis the less the differences become, and at the ultrastructural level all hypertrophied myocardium looks much the same.

\section{References}

Arvidsson, H. (I961). Angiocardiographic determination of left ventricular volume. Acta Radiologica, 56, 321 .

Coyne, J. J. (1968). New concepts of intramural myocardial conduction in hypertrophic obstructive cardiomyopathy. British Heart fournal, 30, 546.

Croxson, R. S., and Raphael, M. J. (1969). Angiographic assessment of congestive cardiomyopathy. (Abstr.). British Heart fournal, 31, 390.

Gau, G., Goodwin, J. F., Oakley, C. M., Raphael, M. J., and Steiner, R. E. (1970). $Q$ waves and coronary angiography in cardiomyopathy. (Abstr.) British Heart fournal, 32, 554.

Goodwin, J. F. (1970). Congestive and hypertrophic cardiomyopathies. Lancet, $\mathrm{x}, 73 \mathrm{I}$.

- and Oakley, C. M. (1970). The importance of the left ventricular end-diastolic pressure in hypertrophic and obstructive cardiomyopathy. To be published.
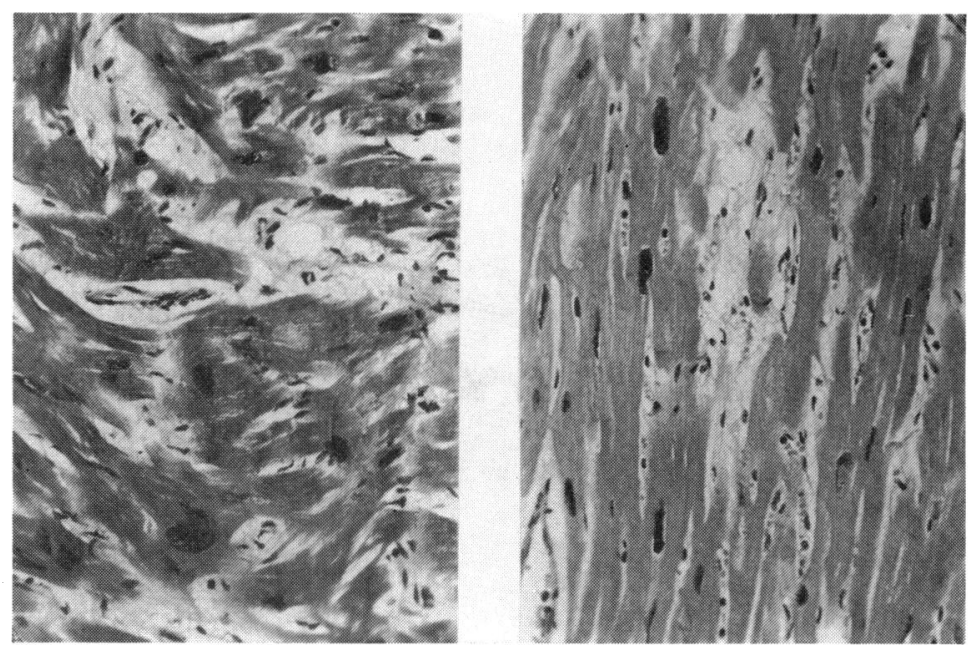

FIG. 7 Conventional light microscopy in hypertrophic cardiomyopathy (left) shows bizarre disarrangement of the fibres, which are abnormally short and wide compared with the hypertrophied fibres from a patient with congestive cardiomyopathy (right), in which the appearances closely resemble those of hypertrophied myocardium due to any cause.

Kristinsson, A. (1969). The congestive cardiomyopathies. $\mathrm{Ph} . \mathrm{D}$. Thesis, University of London.

—, Van Noorden, S., Olsen, E. G. J., Goodwin, J. F., McDonald, L., Oakley, C. M., and Somerville, J. (1969). Hypertrophy and obstruction of the outflow tract of the left ventricle. (Abstr.) British Heart fournal, 32, 554.

Oakley, C. M., Raftery, E. B., Brockington, I. F., Steiner, R. E., and Goodwin, J. F. (1967). Relation of hypertrophic obstructive cardiomyopathy to subvalvar mitral incompetence. (Abstr.) British Heart fournal, 29, 629.

Pridie, R. B., and Oakley, C. M. (1970). Mechanism of mitral regurgitation in hypertrophic obstructive cardiomyopathy. British Heart fournal, 32, 203.

Rackley, C. E., Dodge, H. T., Coble, Y. D., Jr., and Hay, R. E. (1964). A method for determining left ventricular mass in man. Circulation, 29, 666.

Raftery, E. B., Banks, D. C., and Oram, S. (1969). Occlusive disease of the coronary arteries presenting as primary congestive cardiomyopathy. Lancet, 2, II47.

Webb-Peploe, M., Croxson, R. S., and Oakley, Celia (1971). Beta adrenergic blockade with practolol in hypertrophic obstructive cardiomyopathy. (Abstr.) British Heart Fournal, 33, I43.

Wigle, E. D. (1964). Muscular subaortic stenosis: the clinical syndrome, with additional evidence of ventricular septal hypertrophy. In The Cardiomyopathies (Ciba Foundation Symposium), p. 49. Ed. by G. E.W. Wolstenholme and M. O'Connor. Churchill, London. 